\title{
Structuralizing Disaster-scene Data through Auto-captioning
}

\author{
Alina Klerings \\ Ruprecht-Karls-UniversitÃdt \\ Heidelberg \\ Heidelberg, Germany \\ klerings@cl.uni-heidelberg.de
}

\author{
Shiming Tang \\ University of Missouri-Kansas City \\ Kansas City, USA \\ st78d@mail.umkc.edu
}

\author{
ZhiQiang Chen* \\ chenzhiq@umkc.eduy \\ University of Missouri-Kansas City \\ Kansas City, MO
}
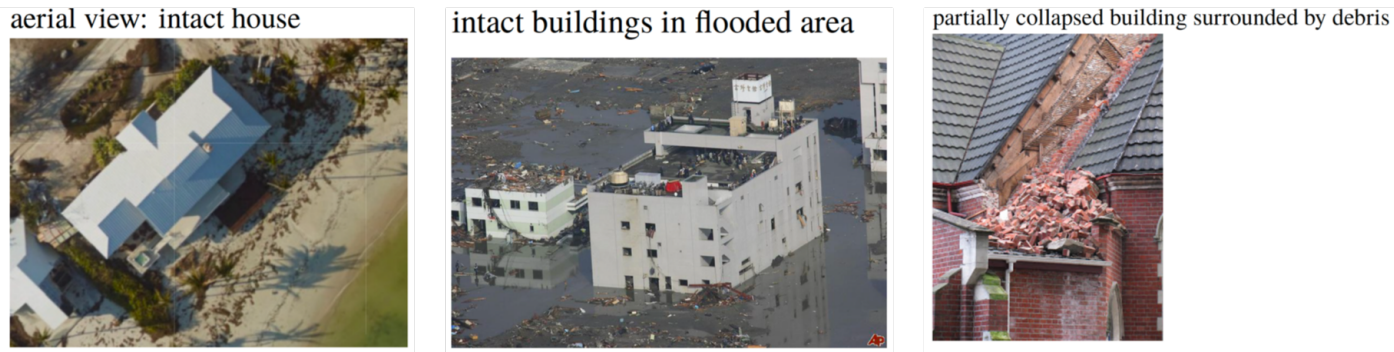

Figure 1: Captioned disaster-scene images.

\begin{abstract}
Disaster-scene images documenting the magnitude and effects of natural disasters nowadays can be easily collected through crowdsourcing aided by mobile technologies (e.g., smartphones or drones). One challenging issue that confronts the first-responders who desire the use of such data is the non-structured nature of these crowdsourced images. Among other techniques, one natural way is to structuralize disaster-scene images through captioning. Through captioning, their imagery contents are augmented by descriptive captions that further enable more effective search and query (S\&Q). This work presents a preliminary test by exploiting an end-to-end deep learning framework with a linked CNN-LSTM architecture. Demonstration of the results and quantitative evaluation are presented that showcase the validity of the proposed concept.
\end{abstract}

\section{CCS CONCEPTS}

- Information systems $\rightarrow$ Information systems applications; Spatial-temporal systems; Location based services; • Applied computing $\rightarrow$ Physical sciences and engineering; Engineering;

\section{KEYWORDS}

Disaster scenes, Deep learning, Autonomous caption, Disaster resilience

${ }^{*}$ Corresponding author

Permission to make digital or hard copies of all or part of this work for personal or classroom use is granted without fee provided that copies are not made or distributed for profit or commercial advantage and that copies bear this notice and the full citation on the first page. Copyrights for components of this work owned by others than ACM must be honored. Abstracting with credit is permitted. To copy otherwise, or republish, to post on servers or to redistribute to lists, requires prior specific permission and/or a fee. Request permissions from permissions@acm.org.

ARIC'19, November 5-8, 2019, Chicago, IL, USA

(c) 2019 Association for Computing Machinery.

ACM ISBN 978-1-4503-6954-1/19/11 ..\$15.00

https://doi.org/10.1145/3356395.3365671
ACM Reference Format:

Alina Klerings, Shiming Tang, and ZhiQiang Chen. 2019. Structuralizing Disaster-scene Data through Auto-captioning. In 2nd ACM SIGSPATIAL International Workshop on Advances on Resilient and Intelligent Cities (ARIC'19), November 5-8, 2019, Chicago, IL, USA. ACM, New York, NY, USA, 4 pages. https://doi.org/10.1145/3356395.3365671

\section{INTRODUCTION}

Severe disasters often trigger significant economic loss and human lives that overwhelm the capacity of civic communities and industrial sectors. Resilient disaster response, including preparation and recovery, is of paramount importance to urban and rural communities. On the other hand, disaster scenes from extreme events, such as hurricanes, storms, surges, earthquakes, tsunamis, and tornadoes, are extremely complex at different scales. At a ground level, it visually manifests as dynamic and complex appearances subject to different disasters. Disaster scenes are perishable in nature due to the organized recovery efforts of society. Therefore, 'learningfrom-disasters' efforts, including data collection, interpretation, and mapping, are essential in order to obtain critical informatics for rapid disaster response. For data collection, the traditional platforms include space- or airborne remote sensors, e.g., satellite or aircraft based earth-observation imagers. For example, the modern satellite sensors, such as the Worldview-1 to 4 series, are available to provide multi-temporal imagery at very high resolution. In recent years, the emerging trend is community-based or personalized mobile remote sensing technologies that become more abundant as mobile devices become ubiquitous, in general, which can be termed as crowdsourcing based remote sensing. These technologies include the use of small unmanned aerial vehicles (UAVs or colloquially called drones) (e.g., [9]) and the use of smartphones and other smart mobile devices. This technology abundance alleviates significantly the stress of collecting perishable disaster data [1,2]. Therefore, crowdsourcing has been recognized by government agencies as a useful information technology approach [11]. 
On the other hand, the use of these emerging technologies results in big data, mostly non-structured imagery data in nature can significantly burden and discourage the first responders toward embracing the crowdsourcing approach. Among many structuralization techniques, including the traditional database-driven methods, such as the use of simple attributes or relations, including location, time stamps, authors, event/occasion, etc., advanced techniques are being explored. A recent effort includes the use of deep transfer learning methods to classify the disaster-scene images [13]. In the deep learning community, an emerging approach is automatic captioning [3]. Automatic captioning for a large volume of images has significant value that is beyond the traditional database-driven attributes-based search and query (S\&Q). With captions, one can conduct more effective content-based image retrieval (CBIR). For example, in a disaster-response context, a first-responder can ask for images within a certain geographical boundary that are related to severely damaged residence houses, instead of multiple $S \& Q$ steps to reach the desired results. In this work, we hypothesize that building upon modern deep learning-based models, disaster-scene images can be captioned automatically with acceptable accuracy towards a meaningful implementation in the near future. To proceed, this work presents a deep learning approach to auto-generate captions for disaster scene pictures given a small set of training images with manual captions.

\section{METHODOLOGY}

\subsection{Related Work}

One challenge that pertains to image captioning is the correct extraction of relations between the extracted objects. To produce descriptions that are not only meaningful but also grammatically correct, the model needs to learn natural language from the training captions. Therefore, to process the images together with their captions, an encoder-decoder architecture was utilized, consisting of a convolutional neural network ( $\mathrm{CNN})$ and a long short-term memory recurrent neural network (LSTM). This model setup is an adaption of the Show-and-Tell Model [14] that was inspired by sequence modeling approaches in machine translation. This work presents an end-to-end-system for generating descriptive captions for disaster scene images. It also includes a data set of roughly 2000 pictures of earthquake and tornado results, labeled with descriptions. To the knowledge of the authors, there is no application of this model (or similar automatic captioning models, e.g., [4]) in the domain of disaster-scene understanding.

Tackling the challenge of image captioning with linked neural networks is not a new approach. [5] introduced an architecture very similar to the one presented in this work. They utilized an alignment model consisting of a $\mathrm{CNN}$ for image regions, that learns to align visual and language data, and a bidirectional recurrent neural network for sequence learning. The main differences from the Show-and-Tell model [14] are the pre-trained word2vec weights for the embedding space, a bias term to incorporate the image information and the RNN which is less complex than the utilized LSTM here.

The Show-and-Tell Model uses an image data set with captions as the training input, with two-piece features consisting of an image part and a caption part. However, one image, together with its caption, does not make for one single data point but rather multiple data points, depending on the caption length. Since the caption is predicted word by word, a data point consists of an image and a partial caption as expressed as follows. $x$ is the encoded image + word embedding of a partial caption; and $y$ is the predicted word, next in the sequence of a partial caption.

\subsection{Disaster Scene Data Set}

The disaster-scene data set which is part of the contribution of this work consists of 2003 images from two earthquake events (the 2011 Christchurch and the 2011 Great East Japan) and two recent data collection efforts from the 2019 Missouri and Kansas tornadoes. The recent Tornado data was obtained from the mobile platform Fulcrum, supported by a National Science Foundation-funded effort [8].

For the earthquake datasets, trained student assistants manually generated the caption using a minimal number of words with a complete disaster-scene description (e.g., the primary object type, the hazard type, the damage level, or other prominent attributes in the images). For the tornado disasters, it is noted that a descriptive caption for each image was automatically retrieved from the database created by the Fulcrum smart app. In addition, the captions were manually checked for spelling mistakes and relevance and, if necessary, updated. Because of the small size of the data set, noise in the form of incorrect and inaccurate descriptions had to be prevented at all costs. As a result, in this effort, we achieve the training and testing data sets: 1803 images with captions for training, 100 images with captions for model verification set, and another 100 images for human-based testing and validation.

\subsection{Model Adaption and Training}

The original Show-and-Tell Model was employed for the comprehensive COCO data set [7]. For the adaption to the specific disaster scene domain, most models and training settings could be inherited. A stochastic gradient descent optimizer for the LSTM was applied with an input and output embedding size of 512. The word embeddings were not initialized from a bigger corpus because that did not improve the results and only complicated the architecture [14].

Since the domain-specific training set is significantly smaller than the original database, a preprocessing adjustment was necessary: All words of the captions were added to the vocabulary, as long as they occurred at least 3 times (as opposed to a minimum word count of 5 for the bigger COCO data set). The remaining words were labeled with "UNKNOWN" during tokenization. The original Show-and-Tell model was trained based on the COCO dataset. Instead of enriching the COCO data set with disaster scene images and re-training the original neural network, which would add the ability to deal with a new domain to the already strong model, this work presents a transfer-learning based approach.

By applying transfer learning, the disaster scene input pictures are encoded using the Inception v3 model [12], a pre-trained CNN, commonly utilized for image tasks. The last Softmax layer of the $\mathrm{CNN}$ is removed, so it works as a static image encoder function. That means the CNN does not classify the images into one of the 1000 ImageNet classes but extracts a fixed-length vector, the encoded image. This process of utilizing the last hidden layer of a 
neural network to extract bottleneck training features is known as automatic feature engineering [6]. Subsequently, a single transformation layer maps the $\mathrm{CNN}$ output into the word embedding space.

To process the captions of the training set, a vocabulary of all unique words with an individual index is generated, including an additional START and END token. Every partial caption is transformed into a sequence of words and then to a sequence of indices with the size of the maximum caption length, if necessary, using zero paddings. To utilize the sequences as caption features in form of fixed-length vector representations, word embeddings are created that are learned for each word in the vocabulary during training.

During the initial training phase with the combined image and caption feature vectors, the parameters of the transformation layer, the parameters of the word embeddings, and the weights of the LSTM are trained. The LSTM functions as a sequence-generating language model conditioned on the image encoding, which is used to set up its initial state. The image feature vector and the word embeddings of the partial caption are employed to predict the probability distributions for the next word. It is noted that the output of the LSTM at time t- 1 is fed to the LSTM at time $t$, meaning that the preceding words are always considered for the prediction of the next word, which is decoded from its vector representation into natural language. In a second phase, all parameters from the initial training plus the weights of the $\mathrm{CNN}$ are fine-tuned jointly.

At inference time, test images are encoded and incorporated as the first word to generate a caption sequence. For that, beam search is applied, which - as opposed to greedy search - returns a list of the $\mathrm{k}$ most likely output sequences by expanding all possible next steps. Naturally, a higher beam size $\mathrm{k}$ yields more output sentences and should, therefore, increase the chances for a good caption. However, experiments with different values for $\mathrm{k}$ show that the performance for $\mathrm{k}=3$ instead of $\mathrm{k}=20$ improves the BLEU4 score by 2 points on the $\mathrm{COCO}$ data set. The authors presume that their model has overfitted and therefore keep the low beam size for regularization to introduce a little extra noise.

\section{RESULTS AND VALIDATION}

\subsection{Results}

Auto-generated captions are tabulated as shown in Table 1. Amid the results, we observed the obtained auto-captioning model through the transfer learning mechanism show promising results. A large percentage of the captions describe the images without errors. Some have minor errors; some show a description related to the image; it is found too that some captions are mistaken about the contents of the images.

\subsection{Performance Validation}

Image captioning is a natural language processing task and, therefore, not as easy to evaluate as a simple classification problem. A standard measure for sequence modeling like machine translation is the BLEU score, which compares a generated sentence against one or more reference sentences [10]. It was utilized for this work because it is easy to compute and correlates with human evaluation. Table 2 and Table 3 presents the BLEU scores on the test data, while the two results used different model fine-tuning steps.
Training on the sparse disaster scene data makes the model prone to overfitting, an issue that was already faced with the much larger COCO data set. Several measures to prevent overfitting like reducing the beamsize, dropout, and freezing the $\mathrm{CNN}$ weights during initial training have already been employed for the original model. For the disaster scene data, the number of steps and learning rate were modified. A first training phase with 10,000 steps and an initial learning rate of 0.5 to update the weights of LSTM, word embeddings, and transformation layer was followed by a second fine-tuning phase to train all model parameters jointly. The performance was tested with an additional 7,700 and 90,000 finetuning steps with a learning rate of 0.0005 , respectively. The latter increases the risk of overfitting, BLEU scores were much higher, possibly indicating that the model already overfitted.

In addition, a human evaluation following the rating system of the Show-and-Tell paper is provided in Table 4, was conducted, where all generated captions for the test set were rated by a human. It indicated the very similar performance of both models, though the longer trained system tended to make more grammar errors, which included word duplicates or incorrect grammatical structures. Furthermore, experiments with different beam sizes indicated the same trends as in [15], so it was kept relatively small for some extra noise during inference $(\mathrm{k}=3)$, which led to an improvement of several BLEU-4 points compared to $\mathrm{k}=20$. For the BLEU scores, averaged over the test set, see tables 2 and 3 .

\subsection{Conclusions and Recommendation}

This paper proposes an automatic caption technique for disasterscene images âĂ $\breve{T}$ preliminary research through the use of an endto-end deep learning framework (a linked CNN-LSTM architecture). Quantitative performance evaluation is presented that showcases the validity of the proposed concept. When looking at some examples of generated captions, it can be seen that the captions are semantically simple. In this paper, the captions used in the training data have a total of 322 different words, which is indeed a small vocabulary. Nonetheless, the model serves its purpose and generates informative descriptions for disaster scene images. To further improve the performance, more training data could be added to prevent overfitting while enabling better tuning of the model parameters. Performance evaluation (e.g., computational time) at different devices should be evaluated as well.

In the future, the presented end-to-end caption generation system could be incorporated into a smart application, possibly combined with other classification techniques that return much accurate recognition of disaster types and damage degree. The authors envision that with a future version of this system, the first responders who desire the use of crowdsourced images for decision-making may work in two different ways. The first, given a regional perimeter, the user may query in terms of all buildings that are damaged (at a certain level) in this region that are reported by the crowd, then all images with relevant captions will be showcased. Second, in a much-augmented version, we further imagine that the end-users working in the field can interact with his or her mobile devices by cognitive voice-based query, and the system then returns images with semantically rich and similar captions for the user's advanced decision making. 
Table 1: Examples of auto-generated captions.

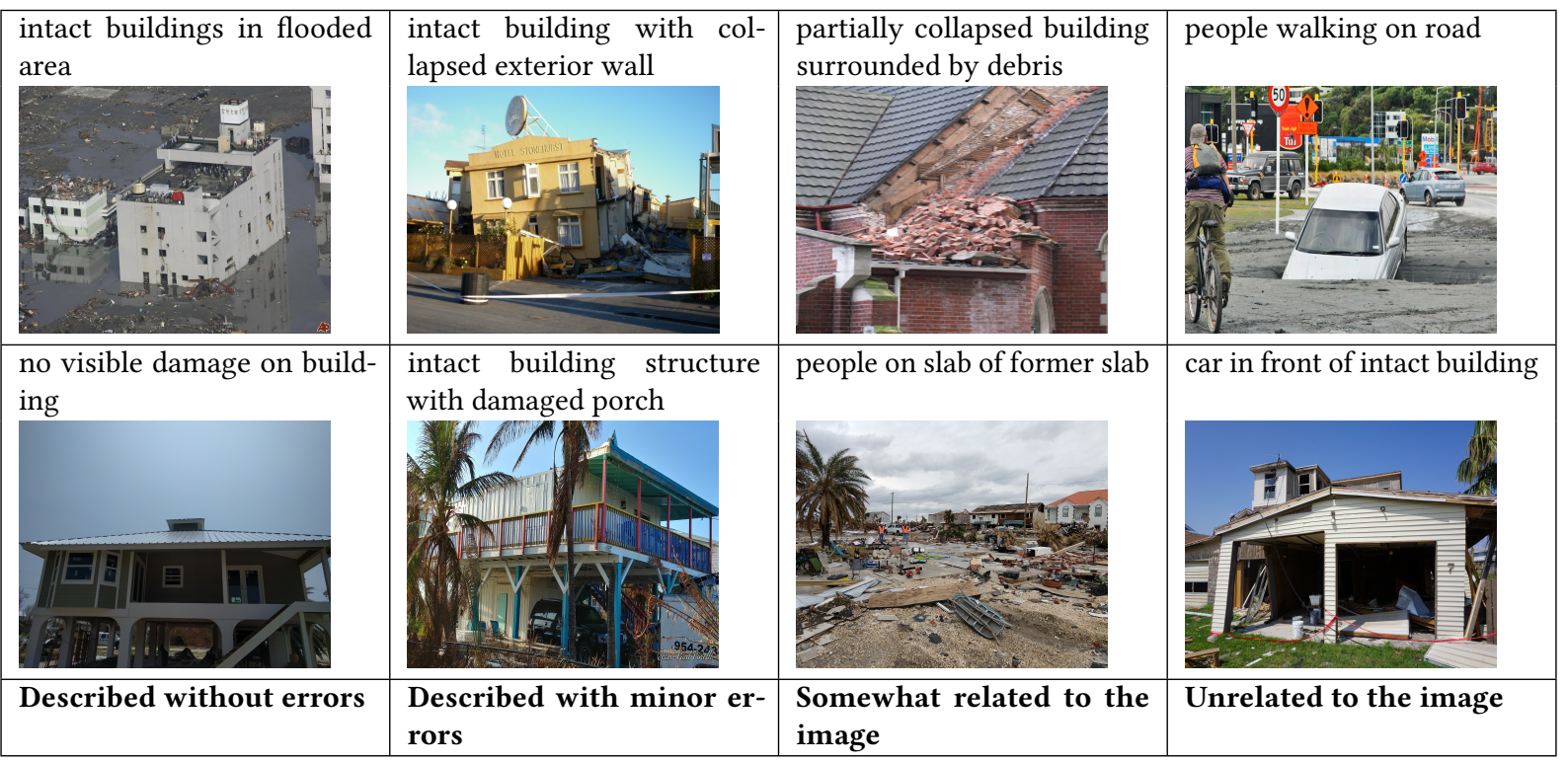

Table 2: BLEU scores on test set of disaster scene data, 7,700 fine-tuning steps, model perplexity: 9.9

\begin{tabular}{l|l|l|l|l} 
& BLEU-1 & BLEU-2 & BLEU-3 & BLEU-4 \\
\hline $\mathbf{k}=\mathbf{3}$ & 47.7 & 36.6 & 32.5 & 27.3 \\
\hline $\mathbf{k}=\mathbf{2 0}$ & 47.8 & 36.0 & 31.7 & 26.3
\end{tabular}

Table 3: BLEU scores on test set of disaster scene data, 90k fine-tuning steps, model perplexity: 10.1

\begin{tabular}{l|l|l|l|l} 
& BLEU-1 & BLEU-2 & BLEU-3 & BLEU-4 \\
\hline $\mathbf{k = 3}$ & 53.0 & 41.3 & 30.8 & 36.9 \\
\hline $\mathbf{k}=\mathbf{2 0}$ & 50.6 & 39.5 & 35.3 & 29.7
\end{tabular}

Table 4: Human evaluation

100 Test captions generated by model with 7,700 and 90,000 fine-tuning steps respectively

\begin{tabular}{l|l|l|l} 
& Rating & $\mathbf{7 k}$ & $\mathbf{9 0 k}$ \\
\hline $\mathbf{4}$ & describes without errors & 53 & 47 \\
\hline $\mathbf{3}$ & describes with minor errors & 25 & 30 \\
\hline $\mathbf{2}$ & somewhat related to the image & 9 & 11 \\
\hline $\mathbf{1}$ & unrelated to the image & 12 & 11 \\
\hline \hline & Grammar errors & 5 & 13
\end{tabular}

\section{REFERENCES}

[1] D.L. Burton, G. Williams, and R. Williams. 2012. Using Social Media in Disaster Recovery: The foplin Tornado (2011) and Branson Tornado (2012) Model. CreateSpace Independent Publishing Platform, Scotts Valley, CA.

[2] H. Gao, G. Barbier, and R. Goolsby. 2011. Harnessing the crowdsourcing power of social media for disaster relief. Intelligent Systems, IEEE 26, 3 (2011), 10-14.

[3] MD Hossain, Ferdous Sohel, Mohd Fairuz Shiratuddin, and Hamid Laga. 2019. A comprehensive survey of deep learning for image captioning. ACM Computing Surveys (CSUR) 51, 6 (2019), 118.
[4] Andrej Karpathy and Li Fei-Fei. 2015. Deep visual-semantic alignments for generating image descriptions. In Proceedings of the IEEE conference on computer vision and pattern recognition. IEEE, 3128-3137.

[5] Andrej Karpathy and Li Fei-Fei. 2017. Deep Visual-Semantic Alignments for Generating Image Descriptions. IEEE Trans. Pattern Anal. Mach. Intell. 39, 4 (April 2017), 664-676. https://doi.org/10.1109/TPAMI.2016.2598339

[6] Will Koehrsen. 2018. Automated Feature Engineering in Python. https://towardsdatascience.com/automated-feature-engineering-in-python99baf11cc219

[7] Tsung-Yi Lin, Michael Maire, Serge J. Belongie, Lubomir D. Bourdev, Ross B. Girshick, James Hays, Pietro Perona, Deva Ramanan, Piotr Dollár, and C. Lawrence Zitnick. 2014. Microsoft COCO: Common Objects in Context. CoRR abs/1405.0312 (2014). arXiv:1405.0312 http://arxiv.org/abs/1405.0312

[8] StEER network. 2019. NSF Structural Extreme Events Reconnaissance (StEER) Network. https://web.fulcrumapp.com/communities/nsf-rapid

[9] John Oliensis. 2000. A critique of structure-from-motion algorithms. Computer Vision and Image Understanding 80, 2 (2000), 172-214.

[10] Kishore Papineni, Salim Roukos, Todd Ward, and Wei-Jing Zhu. 2002. BLEU: a method for automatic evaluation of machine translation. In Proceedings of the 40th annual meeting on association for computational linguistics. Association for Computational Linguistics, 311-318.

[11] Jesse A Sievers. 2015. Embracing Crowdsourcing: a strategy for state and local governments Approaching âĂIJWhole CommunityâĂİ Emergency Planning. State and Local Government Review 47, 1 (2015), 57-67.

[12] Christian Szegedy, Vincent Vanhoucke, Sergey Ioffe, Jon Shlens, and Zbigniew Wojna. 2016. Rethinking the inception architecture for computer vision. In Proceedings of the IEEE conference on computer vision and pattern recognition. 2818-2826.

[13] Shimin Tang and ZhiQiang Chen. 2019. Machine Understanding of DisasterScene Mechanics. IEEE Journal or Selected Topics in Applied Earth Observations and Remote Sensing (under review) (2019).

[14] Oriol Vinyals, Alexander Toshev, Samy Bengio, and Dumitru Erhan. 2014. Show and tell: A neural image caption generator. 2015 IEEE Conference on Computer Vision and Pattern Recognition (CVPR) (2014), 3156-3164.

[15] Oriol Vinyals, Alexander Toshev, Samy Bengio, and Dumitru Erhan. 2016. Show and Tell: Lessons learned from the 2015 MSCOCO Image Captioning Challenge. IEEE Transactions on Pattern Analysis and Machine Intelligence 39 (07 2016), 1-1. https://doi.org/10.1109/TPAMI.2016.2587640 